\title{
A new formulation of finite difference and finite volume methods for solving a space fractional convection-diffusion model with fewer error estimates
}

\author{
Reem Edwan', Shrideh Al-Omari ${ }^{* *}$ (D), Mohammed Al-Smadi ${ }^{3,4}$, Shaher Momani ${ }^{4,5}$ and Andreea Fulga ${ }^{6}$
}

"Correspondence:

s.k.q.alomari@fet.edu.jo

${ }^{2}$ Department of Physics and Basic

Sciences, Faculty of Engineering

Technology, Al-Balqa Applied

University, Amman, Jordan

Full list of author information is

available at the end of the article

\begin{abstract}
Convection and diffusion are two harmonious physical processes that transfer particles and physical quantities. This paper deals with a new aspect of solving the convection-diffusion equation in fractional order using the finite volume method and the finite difference method. In this context, we present an alternative way for estimating the space fractional derivative by utilizing the fractional Grünwald formula. The proposed methods are conditionally stable with second-order accuracy in space and first-order accuracy in time. Many comparisons are performed to display reliability and capability of the proposed methods. Furthermore, several results and conclusions are provided to indicate appropriateness of the finite volume method in solving the space fractional convection-diffusion equation compared with the finite difference method.
\end{abstract}

Keywords: Finite volume method; Finite difference method; Space fractional convection-diffusion equation; Riemann-Liouville fractional derivative;

Grünwald-Letnikov fractional derivative

\section{Introduction}

The mathematical model is one of the most important logical aspects used to help us explain and study physical phenomena. Many physical situations include non-Markov processes, earthquakes, and terrestrial surface transport can be modeled through a class of diffusion equations or wave equations. In particular, the physical quantities are transferred inside physical systems by diffusion and the flow of heat and stochastically changing systems are described. In recent years, researchers have focused on finding effective numerical methods to solve such a type of partial differential equations (PDEs). Different PDEs have been studied and resolved including the space-time fractional diffusionwave equation [1], the space fractional convection-diffusion equation [2], the space fractional Cauchy equation [3], the fractional heat- and wave-like equations [4], the fractional Korteweg-de Vries equation [5], and so forth [6-11]. Different effective methods have been proposed by scholars such as the reproducing kernel method [12-15], the residual

(c) The Author(s) 2021. This article is licensed under a Creative Commons Attribution 4.0 International License, which permits use, sharing, adaptation, distribution and reproduction in any medium or format, as long as you give appropriate credit to the original author(s) and the source, provide a link to the Creative Commons licence, and indicate if changes were made. The images or other third party material in this article are included in the article's Creative Commons licence, unless indicated otherwise in a credit line to the material. If material is not included in the article's Creative Commons licence and your intended use is not permitted by statutory regulation or exceeds the permitted use, you will need to obtain permission directly from the copyright holder. To view a copy of this licence, visit http://creativecommons.org/licenses/by/4.0/ 
power series method [16-18], the homotopy perturbation method [19], the multi-step approach [20], and some others [21-24].

During the past years, certain numerical methods, such as the finite difference method, the finite volume method, and the finite element method, have been developed to solve some PDEs. The important goal of these methods is to convert the equation to a system of linear algebraic equations. For example, in [25], the time-fractional convection-diffusion equations have been reduced to a system of linear algebraic equations using the Gegenbauer polynomials and Lagrange polynomials space and time expansion, respectively. In [26], Saadatmandi et al. studied the sinc-Legendre collocation technique for solving a class of fractional convection-diffusion equations with variable coefficients. In [27], the finite volume element technique was used to solve the sub-diffusion problems in fractional order. In addition, Jia and Wang used the volume penalization approach to improve the finite volume technique for finding a solution for a space fractional diffusion equation [28]. In [29], Mahto et al. applied the continuation property to propose the approximate solution of the sub-diffusion equation via internal control. In [30], Zhao et al. applied the finite element technique for solving the time-fractional diffusion problem, they introduced an unconditionally stable scheme based on the spatial quasi-Wilson nonconforming; while in [31], the authors derived the optimal error estimates by studying the two mixed finite element methods, conforming and nonconforming, to solve the time-fractional diffusion problem. On the other hand as well, Stynes et al. used a different technique to solve the time-fractional diffusion equation and to find elaborate error boundedness based on the regularity of the exact solution [32]. In [33], Sayevand et al. proposed the finite volume element technique for analyzing the behavior of sub-diffusion equations and proved its stability analysis of obtained solution. In [34], the authors proposed the finite difference discretization based on the weighted trapezoidal rule to approximate the fractional Laplacian and proved the convergence in one dimension. More interesting applications can be found in [35-45].

In this paper, we deal with a new aspect of FVM and FDM to obtain approximate solution for the space fractional convection-diffusion equation (SFCDE), in which the space fractional derivative is represented in the Riemann-Liouville sense. More specifically, let us consider the SFCDE of the following form:

$$
\frac{\partial \psi(x, t)}{\partial t}+\epsilon \frac{\partial^{\beta} \psi(x, t)}{\partial x^{\beta}}=\rho \frac{\partial^{2} \psi(x, t)}{\partial x^{2}},
$$

subject to the initial condition

$$
\psi(x, 0)=g(x), \quad 0 \leq x \leq L,
$$

where $t \geq 0, x \in[0, L]$, a parameter $\beta$ describes the order of the space fractional derivative, $0<\beta \leq 1$, so that the space fractional derivative is described in the Riemann-Liouville sense, $\psi(x, t)$ is concentration, $\epsilon$ and $\rho$ are positive parameters, $\rho$ is a diffusion coefficient, $\epsilon$ is the velocity of water flow, and $L$ is the length of the channel. For $\beta=1$, the equation, indeed, reduces to the linear convection-diffusion equation. In particular, the exact solution of the linear convection-diffusion equation of the shape

$$
\frac{\partial \psi(x, t)}{\partial t}+\epsilon \frac{\partial \psi(x, t)}{\partial x}=\rho \frac{\partial^{2} \psi(x, t)}{\partial x^{2}}
$$


subject to the initial condition $\psi(x, 0)=-\sin (\pi x)$ is given in the form

$$
\psi(x, t)=-\sin (\pi(x-\epsilon t)) e^{-\rho \pi^{2} t} .
$$

The structure of this analysis is as follows. In Sect. 2, some preliminary definitions and theorems related to Grünwald-Letnikov fractional derivative are introduced. Section 3 is devoted to constructing the FDM for FIVPs (1) and (2) along with its theoretical analysis. In Sect. 4, the FVM is proposed to solve FIVPs (1) and (2) along with its theoretical analysis. In Sect. 4, numerical examples are given to express validity and reliability of the methods and to make a comprehensive comparison for both FVM and FDM. Finally, some conclusions are given in Sect. 5.

\section{Basic definitions}

In the literature there are numerous integrals which are widely used in physics, astronomy, and engineering $[46,47]$. Consequently, different types of fractional integrals and derivatives have been found very reliable under some conditions. In this section, we introduce some preliminary definitions and theorems related to the Riemann-Liouville fractional derivative and Grünwald-Letnikov fractional derivative.

Definition 1 The Riemann-Liouville integral of fractional order $\alpha>0, J_{a}^{\alpha} \psi(x)$, is defined as

$$
J_{a}^{\alpha} \psi(x)=\frac{1}{\Gamma(\alpha)} \int_{a}^{x}(x-t)^{\alpha-1} \psi(t) d t, \quad \text { provided that } \psi \in L_{1}[a, b] .
$$

For $\alpha=0$, the Riemann-Liouville integral of fractional order $J_{a}^{0} \psi(x)=\psi(x)$ is the identity operator.

Definition 2 Let $n$ be the smallest integer that exceeds $\alpha$, then the Riemann-Liouville fractional derivative of order $\alpha>0$ is defined as

$$
\begin{aligned}
\mathcal{D}_{a}^{\alpha} \psi(x) & =\mathcal{D}^{n} J_{a}^{(n-\alpha)} \psi(x) \\
& =\frac{1}{\Gamma(n-\alpha)}\left(\frac{d^{n}}{d x^{n}}\right)\left[\int_{a}^{x} \frac{\psi(t)}{(x-t)^{\alpha+1-n}} d t\right] .
\end{aligned}
$$

For $\alpha \in \mathbb{N}$, the Riemann-Liouville fractional derivative of order $\alpha$ is given as $\mathcal{D}_{a}^{\alpha} \psi(x)=$ $\frac{d^{\alpha} \psi(x)}{d x^{\alpha}}$.

Definition 3 Let $\psi \in C^{[\alpha]}[a, b], \alpha>0$. Then the Grünwald-Letnikov fractional derivative of the function $\psi$ of order $\alpha$ is given by

$$
\tilde{\mathcal{D}}_{a}^{\alpha} \psi(x)=\lim _{h \rightarrow 0} \frac{1}{h^{\alpha}} \sum_{k=0}^{\left[\frac{x-a}{h}\right]}(-1)^{k}\left(\begin{array}{l}
\alpha \\
k
\end{array}\right) \psi(x-k h), \quad a<x \leq b,
$$

where $h=\frac{x-a}{N}$.

The following theorem shows the relation between this definition and the RiemannLiouville fractional derivatives. 
Theorem 1 Let $\alpha>0, n=\lceil\alpha\rceil$ and $\psi \in C^{n}[a, b]$. Then we have

$$
\tilde{\mathcal{D}}_{a}^{\alpha} \psi(x)=\mathcal{D}_{a}^{\alpha} \psi(x), \quad a<x \leq b .
$$

Theorem 2 Let $\alpha>0$ and $\psi \in C[a, b]$. Then we have

$$
J_{a}^{\alpha} \psi(x)=\lim _{h \rightarrow 0} h^{\alpha} \sum_{k=0}^{\left[\frac{x-a}{h}\right]}(-1)^{k}\left(\begin{array}{c}
-\alpha \\
k
\end{array}\right) \psi(x-k h), \quad h=\frac{x-a}{N}, a<x \leq b,
$$

where $(-1)^{k}\left(\begin{array}{c}-\alpha \\ k\end{array}\right)=\frac{\alpha(\alpha-1)(\alpha-2) \ldots(\alpha-k+1)}{k !}=\frac{\Gamma(\alpha+k)}{\Gamma(\alpha) \Gamma(k+1)}$ and $\Gamma(x)$ is the gamma function given by $\Gamma(x)=\int_{0}^{\infty} s^{x-1} e^{-s} d s$. If the weights are given by $v_{0}^{\alpha}=1, v_{1}^{\alpha}=\alpha$ and $v_{k}^{\alpha}=\left(1-\frac{(1-\alpha)}{k}\right) v_{k-1}^{\alpha}, k=$ $2,3, \ldots$, then Eq. (5) may be rewritten as

$$
J_{a}^{\alpha} \psi(x)=\lim _{h \rightarrow 0} h^{\alpha} \sum_{k=0}^{\left[\frac{x-a}{h}\right]} v_{k}^{\alpha} \psi(x-k h), \quad h=\frac{x-a}{N} .
$$

The above formula is used to approximate the fractional integral $J_{a}^{\alpha} \psi(x)$.

Lemma 1 Let $0<\alpha<1$. Then we have

1. $v_{0}^{\alpha}=1$ and $v_{k}^{\alpha}>0$ for $k=1,2, \ldots$.

2. $v_{k}^{\alpha}-v_{k+1}^{\alpha}>0$ for $=0,1, \ldots$.

3. $\lim _{k \rightarrow \infty} v_{k}^{\alpha}=0$.

Proof 1 . By induction and the recursive definition, we indeed have $v_{0}^{\alpha}=1, v_{1}^{\alpha}=\alpha>0$, and

$$
v_{k}^{\alpha}=\left(1-\frac{(1-\alpha)}{k}\right) v_{k-1}^{\alpha}, \quad k=2,3, \ldots
$$

Since $0<\alpha<1$, we have $0<\frac{1-\alpha}{k}<\frac{1}{k}<1$ for $k \geq 2$. Therefore, the coefficient $\left(1-\frac{(1-\alpha)}{k}\right)$ in Eq. (7) strictly lies between zero and one.

2. For $k \geq 2$, we have

$$
\begin{aligned}
v_{k}^{\alpha}-v_{k+1}^{\alpha} & =\left(1-\frac{(1-\alpha)}{k}\right) v_{k-1}^{\alpha}-\left(1-\frac{(1-\alpha)}{k+1}\right) v_{k}^{\alpha} \\
& =\left(1-\frac{(1-\alpha)}{k}\right) v_{k-1}^{\alpha}-\left(1-\frac{(1-\alpha)}{k+1}\right)\left(1-\frac{(1-\alpha)}{k}\right) v_{k-1}^{\alpha} \\
& =\left[1-\left(1-\frac{(1-\alpha)}{k+1}\right)\right]\left(1-\frac{(1-\alpha)}{k}\right) v_{k-1}^{\alpha} \\
& =\frac{(1-\alpha)}{k+1}\left(1-\frac{(1-\alpha)}{k}\right) v_{k-1}^{\alpha} .
\end{aligned}
$$

The result is, therefore, positive. Part 3 is straightforward. Hence, we omit the details. This completes the proof of the lemma. 


\section{Finite difference scheme formulation}

In this section, we propose a new finite difference scheme formulation (FDSF) for solving the SFCDE with constant coefficients

$$
\frac{\partial \psi(x, t)}{\partial t}+\epsilon \frac{\partial^{\beta} \psi(x, t)}{\partial x^{\beta}}=\rho \frac{\partial^{2} \psi(x, t)}{\partial x^{2}}
$$

subject to the initial condition

$$
\psi(x, 0)=g(x), \quad a \leq x \leq b,
$$

in which $t \geq 0,0<\beta \leq 1, g(x)$ is an analytical smooth function of a spatial $x, \psi(x, t)$ is an unknown analytical function, $\epsilon$ and $\rho$ are positive parameters, and $\beta$ is a parameter that describes the order of the space fractional, where the space fractional derivative is described in the Riemann-Liouville sense. If $\beta=1$, then the equation becomes a linear convection-diffusion equation. Now, by utilizing Definition 2 and Riemann-Liouville fractional derivative, we have

$$
\frac{\partial \psi(x, t)}{\partial t}+\epsilon \frac{\partial}{\partial x} J_{a}^{1-\beta} \psi(x, t)=\rho \frac{\partial^{2} \psi(x, t)}{\partial x^{2}}
$$

where $J_{a}^{1-\beta}$ is the Riemann-Liouville integral with respect to $x$. If $\alpha=1-\beta$, then we have $0 \leq \alpha<1$. Define $t_{n}=n \tau, n=0,1, \ldots, M ; x_{i}=a+i h, i=0,1, \ldots, N$, where $\tau$ is the time step and $h$ is the spatial step. Then, we will be using the standard Grünwald formula to approximate the $\alpha$ order fractional Riemann-Liouville integral and the central difference formula to approximate the first and the second derivative as follows:

$$
\begin{aligned}
& J_{a}^{\alpha} \psi(x, t)=h^{\alpha} \sum_{j=0}^{N} v_{j}^{\alpha} \psi(x-j h, t)+o(1), \\
& \left.\frac{\partial \psi(x, t)}{\partial x}\right|_{x=x_{i}}=\frac{\psi\left(x_{i+1}, t\right)-\psi\left(x_{i-1}, t\right)}{2 h}+\mathcal{O}\left(h^{2}\right), \\
& \left.\frac{\partial^{2} \psi(x, t)}{\partial x^{2}}\right|_{x=x_{i}}=\frac{\psi\left(x_{i-1}, t\right)-2 \psi\left(x_{i}, t\right)+\psi\left(x_{i+1}, t\right)}{h^{2}}+\mathcal{O}\left(h^{2}\right) .
\end{aligned}
$$

The starting point for applying the finite difference method by evaluating Eq. (10) at $x=x_{i}$ and using the above equations is that

$$
\begin{aligned}
\frac{d \psi\left(x_{i}, t\right)}{d t}= & -\frac{\epsilon}{2 h}\left[h^{\alpha} \sum_{j=0}^{i+1} v_{j}^{\alpha} \psi\left(x_{i-j+1}, t\right)-h^{\alpha} \sum_{j=0}^{i-1} v_{j}^{\alpha} \psi\left(x_{i-j-1}, t\right)\right] \\
& +\rho\left[\frac{\psi\left(x_{i-1}, t\right)-2 \psi\left(x_{i}, t\right)+\psi\left(x_{i+1}, t\right)}{h^{2}}\right] .
\end{aligned}
$$

Hence, using the standard backward difference to approximate the temporal derivative in Eq. (14) reveals

$$
\left.\frac{d \psi\left(x_{i}, t\right)}{d t}\right|_{t=t_{n+1}}=\frac{\psi\left(x_{i}, t_{n+1}\right)-\psi\left(x_{i}, t_{n}\right)}{\tau}+\mathcal{O}(\tau) .
$$


Letting $\psi_{i}^{n} \approx \psi\left(x_{i}, t_{n}\right)$ denotes that the numerical solution implies

$$
\begin{aligned}
& \frac{\psi_{i}^{n+1}-\psi_{i}^{n}}{\tau} \\
& \quad=-\frac{\epsilon}{2 h}\left[h^{\alpha} \sum_{j=0}^{i+1} v_{j}^{\alpha} \psi_{i-j+1}^{n+1}-h^{\alpha} \sum_{j=0}^{i-1} v_{j}^{\alpha} \psi_{i-j-1}^{n+1}\right]+\rho\left[\frac{\psi_{i-1}^{n+1}-2 \psi_{i}^{n+1}+\psi_{i+1}^{n+1}}{h^{2}}\right] .
\end{aligned}
$$

By collecting like terms, Eq. (16) can be rewritten as

$$
\frac{\psi_{i}^{n+1}-\psi_{i}^{n}}{\tau}+\sum_{j=0}^{N} b_{i j} \psi_{j}^{n+1}=0
$$

where

$$
b_{i j}= \begin{cases}\frac{\epsilon h^{\alpha-1}\left[v_{i-j+1}^{\alpha}-v_{i-j-1}^{\alpha}\right]}{2}, & j<i-1, \\ \frac{\epsilon h^{\alpha-1}\left[v_{2}^{\alpha}-v_{0}^{\alpha}\right]}{2}-\frac{\rho}{h^{2}}, & j=i-1, \\ \frac{\epsilon h^{\alpha-1} v_{1}^{\alpha}}{2}+\frac{2 \rho}{h^{2}}, & j=i, \\ \frac{\epsilon h^{\alpha-1} v_{0}^{\alpha}}{2}-\frac{\rho}{h^{2}}, & j=i+1, \\ 0, & j>i+1 .\end{cases}
$$

Denoting the numerical solution vector by $\psi^{n}=\left[\psi_{0}^{n}, \psi_{1}^{n}, \ldots, \psi_{N}^{n}\right]$, the vector equation becomes

$$
(I+\tau A) \psi^{n+1}=\psi^{n}
$$

where $A$ is a matrix with elements $a_{i j}=b_{i j}$. Hence, the above given equation can be formatted as

$$
\psi^{n+1}=M \psi^{n}
$$

where $M=(I+\tau A)^{-1}$ is the iteration matrix.

The next theorems show that the finite difference scheme FDS expressed in Eq. (20) is conditionally stable and consistent with first-order accurate in time and second-order accurate in space.

We commence to prove the strictly diagonally dominant property for the iteration ma$\operatorname{trix} M$.

Theorem 3 Let $\epsilon>0, \rho>0$, and $0 \leq \alpha<1$ satisfy $\rho>\frac{\epsilon h^{\alpha+1}}{2}$, where $h$ is the spatial step. Then the coefficients $a_{i j}$ satisfy the inequality

$$
\left|a_{i i}\right|>\sum_{\substack{j=0 \\ j \neq i}}^{N}\left|a_{i j}\right|, \quad i=1,2, \ldots, N
$$


Proof Let $i=1,2, \ldots, N$ be given. Then consider the sum

$$
\begin{aligned}
\sum_{\substack{j=0 \\
j \neq i}}^{N}\left|a_{i j}\right| & =\sum_{j=0}^{i-2}\left|a_{i j}\right|+\left|a_{i, i-1}\right|+\left|a_{i, i+1}\right|+\sum_{j=i+2}^{N}\left|a_{i j}\right| \\
& =\sum_{j=0}^{i-2}\left|\frac{\epsilon h^{\alpha-1}\left[v_{i-j+1}^{\alpha}-v_{i-j-1}^{\alpha}\right]}{2}\right|+\left|\frac{\epsilon h^{\alpha-1}\left[v_{2}^{\alpha}-v_{0}^{\alpha}\right]}{2}-\frac{\rho}{h^{2}}\right|+\left|\frac{\epsilon h^{\alpha-1} v_{0}^{\alpha}}{2}-\frac{\rho}{h^{2}}\right| .
\end{aligned}
$$

Therefore, the previous lemma and the hypotheses $\rho>\frac{\epsilon h^{\alpha+1}}{2}$ guarantee that each term is negative. So, we have

$$
\sum_{\substack{j=0 \\ j \neq i}}^{N}\left|a_{i j}\right|=\sum_{j=0}^{i-2} \frac{\epsilon h^{\alpha-1}\left[v_{i-j-1}^{\alpha}-v_{i-j+1}^{\alpha}\right]}{2}+\left(\frac{\rho}{h^{2}}-\frac{\epsilon h^{\alpha-1}\left[v_{2}^{\alpha}-v_{0}^{\alpha}\right]}{2}\right)+\left(\frac{\rho}{h^{2}}-\frac{\epsilon h^{\alpha-1} v_{0}^{\alpha}}{2}\right)
$$

Replacing the finite sum with the infinite sum yields

$$
\sum_{\substack{j=0 \\ j \neq i}}^{N}\left|a_{i j}\right|<\sum_{j=-\infty}^{i-2} \frac{\epsilon h^{\alpha-1}\left[v_{i-j-1}^{\alpha}-v_{i-j+1}^{\alpha}\right]}{2}+\left(\frac{\rho}{h^{2}}-\frac{\epsilon h^{\alpha-1}\left[v_{2}^{\alpha}-v_{0}^{\alpha}\right]}{2}\right)+\left(\frac{\rho}{h^{2}}-\frac{\epsilon h^{\alpha-1} v_{0}^{\alpha}}{2}\right) .
$$

Therefore, the telescoping sum has the form $\left(v_{1}^{\alpha}-v_{3}^{\alpha}\right)+\left(v_{2}^{\alpha}-v_{4}^{\alpha}\right)+\left(v_{3}^{\alpha}-v_{5}^{\alpha}\right)+\left(v_{4}^{\alpha}-v_{6}^{\alpha}\right)$. Now, b y Lemma 1 , this equals $v_{1}^{\alpha}+v_{2}^{\alpha}$. Hence, we have obtained

$$
\begin{aligned}
\sum_{\substack{j=0 \\
j \neq i}}^{N}\left|a_{i j}\right| & <\frac{\epsilon h^{\alpha-1}\left[v_{1}^{\alpha}+v_{2}^{\alpha}\right]}{2}+\left(\frac{\rho}{h^{2}}-\frac{\epsilon h^{\alpha-1}\left[v_{2}^{\alpha}-v_{0}^{\alpha}\right]}{2}\right)+\left(\frac{\rho}{h^{2}}-\frac{\epsilon h^{\alpha-1} v_{0}^{\alpha}}{2}\right) \\
& =\frac{\epsilon h^{\alpha-1} v_{1}^{\alpha}}{2}+\frac{2 \rho}{h^{2}}=\left|a_{i i}\right| .
\end{aligned}
$$

This completes the proof of the theorem.

Corollary 1 The iteration matrix $M$ in Scheme (20) is convergent, so the scheme itself is conditionally stable.

Proof By the previous theorem, we infer that $A$ is strictly diagonally dominant with positive diagonal elements, and so is $I+\tau A$. Therefore, the iteration matrix $M=(I+\tau A)^{-1}$ exists, and its spectral radius satisfies

$$
\rho(M)=\rho(I+\tau A)^{-1}=(1+\tau \rho(A))^{-1}<1 .
$$

The proof is therefore completed.

Theorem 4 Numerical Scheme (17) is consistent with second-order accuracy in space and first-order one in time. 
Proof By using Eqs. (11), (12), (13), and (15), we write Eq. (10) at $\left(x_{i}, t_{n+1}\right)$ as follows:

$$
\begin{aligned}
& \frac{\psi_{i}^{n+1}-\psi_{i}^{n}}{\tau}+\mathcal{O}(\tau) \\
& =-\frac{\epsilon}{2 h}\left[h^{\alpha} \sum_{j=0}^{i+1} v_{j}^{\alpha} \psi_{i-j+1}^{n+1}+o(1)-h^{\alpha} \sum_{j=0}^{i-1} v_{j}^{\alpha} \psi_{i-j-1}^{n+1}+o(1)\right] \\
& \quad+\mathcal{O}\left(h^{2}\right)+\rho\left[\frac{\psi_{i-1}^{n+1}-2 \psi_{i}^{n+1}+\psi_{i+1}^{n+1}}{h^{2}}\right]+\mathcal{O}\left(h^{2}\right) .
\end{aligned}
$$

Therefore, we obtain

$$
\left.\frac{\partial \psi\left(x_{i}, t\right)}{\partial t}\right|_{t=t_{n+1}}=-\left.\epsilon \frac{\partial}{\partial x}\left[J_{a}^{1-\beta} \psi\left(x, t_{n+1}\right)\right]\right|_{x=x_{i}}+\left.\rho \frac{\partial^{2} \psi\left(x, t_{n+1}\right)}{\partial x^{2}}\right|_{x=x_{i}},
$$

which, indeed, leads to Eq. (10) at $\left(x_{i}, t_{n+1}\right)$. The proof of the theorem is complete.

Corollary 2 Numerical Scheme (17) is consistent and stable.

By the fundamental theorem of numerical methods for differential equations, the given numerical scheme is convergent.

\section{Finite volume scheme formulation}

In this section, we propose a novel finite volume scheme formulation (FVSF) for solving the SFCDE with constant coefficients

$$
\frac{\partial \psi(x, t)}{\partial t}+\epsilon \frac{\partial^{\beta} \psi(x, t)}{\partial x^{\beta}}=\rho \frac{\partial^{2} \psi(x, t)}{\partial x^{2}},
$$

subject to the initial condition

$$
\psi(x, 0)=g(x), \quad a \leq x \leq b,
$$

where $t \geq 0,0<\beta \leq 1, g(x)$ is an analytical smooth function of spatial $x, \psi(x, t)$ is an unknown analytical function, $\epsilon$ and $\rho$ are positive parameters, and $\beta$ is a parameter that describes the order of the space fractional, where the space fractional derivative is described in the Riemann-Liouville sense. If $\beta=1$, it becomes a linear convection-diffusion equation.

Now, to establish the finite volume scheme, we need to partition the finite domain $\Omega=$ $[a, b]$ to $N+1$ uniformly spaced nodes $x_{i}=a+i h, i=0,1, \ldots, N$, where the spatial step is $h=\frac{b-a}{N}$. Thus, by utilizing the Riemann-Liouville fractional derivative, we have

$$
\frac{\partial \psi(x, t)}{\partial t}+\epsilon \frac{\partial}{\partial x} J_{a}^{1-\beta} \psi(x, t)=\rho \frac{\partial^{2} \psi(x, t)}{\partial x^{2}}, \quad 0<\beta \leq 1,
$$

where $J_{a}^{1-\beta}$ is the Riemann-Liouville integral with respect to $x$. Setting $\alpha=1-\beta$ gives $0 \leq \alpha<1$. Integrating Eq. (23) over the $i$ th control volume $\left[x_{i-\frac{1}{2}}, x_{i+\frac{1}{2}}\right]$ suggests

$$
\int_{x_{i-\frac{1}{2}}}^{x_{i+\frac{1}{2}}} \frac{\partial \psi(x, t)}{\partial t} d x=\int_{x_{i-\frac{1}{2}}}^{x_{i+\frac{1}{2}}} \frac{\partial}{\partial x}\left[-\epsilon \int_{a}^{\alpha} \psi(x, t)+\rho \frac{\partial u(x, t)}{\partial x}\right] d x .
$$


Dividing each side by $h$ gives the standard finite volume discretization

$$
\frac{d \bar{\psi}_{i}(t)}{d t}=\frac{\epsilon}{h}\left[J_{a}^{\alpha} \psi\left(x_{i-\frac{1}{2}}, t\right)-J_{a}^{\alpha} \psi\left(x_{i+\frac{1}{2}}, t\right)\right]+\frac{\rho}{h}\left[\frac{\partial \psi\left(x_{i+\frac{1}{2}}, t\right)}{\partial x}-\frac{\partial \psi\left(x_{i-\frac{1}{2}}, t\right)}{\partial x}\right],
$$

where

$$
\bar{\psi}_{i}(t)=\frac{1}{h} \int_{x_{i-\frac{1}{2}}}^{x_{i+\frac{1}{2}}} \psi(x, t) d x
$$

is the control volume averages of $\psi(x, t)$.

Using the fractionally-shift Grünwald formula to approximate $J_{a}^{\alpha} \psi(x, t)$ yields

$$
J_{a}^{\alpha} \psi(x, t)=\lim h_{h \rightarrow 0}^{\alpha} \sum_{j=0}^{\left[\frac{x-a}{h}+p\right]} \frac{\Gamma(\alpha+j)}{\Gamma(\alpha) \Gamma(j+1)} \psi(x-(j-p) h, t)
$$

in which $h=\frac{x-a}{N}, x \in(a, b]$. Hence, the Riemann-Liouville integral includes the order term

$$
J_{a}^{\alpha} \psi(x, t)=h^{\alpha} \sum_{j=0}^{N+p} v_{j}^{\alpha} \psi(x-(j-p) h, t)+o(1)
$$

Therefore, due to certain needs of the fractional shift $p=\frac{1}{2}$, we write

$$
\begin{aligned}
& \left.J_{a}^{\alpha} \psi(x, t)\right|_{x=x_{i-\frac{1}{2}}}=J_{a}^{\alpha} \psi\left(x_{i-\frac{1}{2}}, t\right)+h^{\alpha} \sum_{j=0}^{i} v_{j}^{\alpha} \psi\left(x_{i-j}, t\right)+o(1), \\
& \left.J_{a}^{\alpha} \psi(x, t)\right|_{x=x_{i+\frac{1}{2}}}=J_{a}^{\alpha} \psi\left(x_{i+\frac{1}{2}}, t\right)+h^{\alpha} \sum_{j=0}^{i+1} v_{j}^{\alpha} \psi\left(x_{i-j+1}, t\right)+o(1),
\end{aligned}
$$

where $v_{0}^{\alpha}=1, v_{1}^{\alpha}=\alpha$, and $v_{j}^{\alpha}=\left(1-\frac{(1-\alpha)}{j}\right) v_{j-1}^{\alpha}, j=2,3, \ldots$ For $\frac{\partial \psi(x, t)}{\partial x}$, use the identity

$$
\frac{\partial \psi\left(x_{i}, t\right)}{\partial x}=\frac{\psi\left(x_{i+1}, t\right)-\psi\left(x_{i-1}, t\right)}{2 h}+\mathcal{O}\left(h^{2}\right)
$$

to write

$$
\begin{aligned}
& \frac{\partial \psi\left(x_{i-\frac{1}{2}}, t\right)}{\partial x} \approx \frac{\psi\left(x_{i+\frac{1}{2}}, t\right)-\psi\left(x_{i-\frac{3}{2}}, t\right)}{2 h}, \\
& \frac{\partial \psi\left(x_{i+\frac{1}{2}}, t\right)}{\partial x} \approx \frac{\psi\left(x_{i+\frac{3}{2}}, t\right)-\psi\left(x_{i-\frac{1}{2}}, t\right)}{2 h} .
\end{aligned}
$$

For Eqs. (31) and (32), use the standard averaging scheme $\psi\left(x_{i+\frac{1}{2}}, t\right) \approx \frac{\left[\psi\left(x_{i}, t\right)+\psi\left(x_{i+1}, t\right)\right]}{2}$ to construct approximations of first derivative in terms of function values at the nodes $x_{j}$ :

$$
\begin{aligned}
& \frac{\partial \psi\left(x_{i-\frac{1}{2}}, t\right)}{\partial x} \approx \frac{1}{4 h}\left[\psi\left(x_{i}, t\right)+\psi\left(x_{i+1}, t\right)-\psi\left(x_{i-2}, t\right)-\psi\left(x_{i-1}, t\right)\right], \\
& \frac{\partial \psi\left(x_{i+\frac{1}{2}}, t\right)}{\partial x} \approx \frac{1}{4 h}\left[\psi\left(x_{i+1}, t\right)+\psi\left(x_{i+2}, t\right)-\psi\left(x_{i-1}, t\right)-\psi\left(x_{i}, t\right)\right] .
\end{aligned}
$$


So, Eq. (25) can be approximated as

$$
\begin{aligned}
\frac{d \bar{\psi}_{i}(t)}{d t}= & \epsilon h^{\alpha-1}\left[\sum_{j=0}^{i} v_{j}^{\alpha} \psi\left(x_{i-j}, t\right)-\sum_{j=0}^{i+1} v_{j}^{\alpha} \psi\left(x_{i-j+1}, t\right)\right] \\
& +\frac{\rho}{4 h^{2}}\left[\psi\left(x_{i-2}, t\right)-2 \psi\left(x_{i}, t\right)+\psi\left(x_{i+2}, t\right)\right]
\end{aligned}
$$

Notice that if $\psi(x, t)$ is a smooth function, then the value of the control volume averages $\bar{\psi}_{i}(t)$ will agree with the value of $\psi(x, t)$ at the midpoint of the interval $\left[x_{i-\frac{1}{2}}, x_{i+\frac{1}{2}}\right]$ to $\mathcal{O}\left(h^{2}\right)$. So, we can rewrite Eq. (35) as

$$
\begin{aligned}
\frac{d \psi\left(x_{i}, t\right)}{d t}= & \epsilon h^{\alpha-1}\left[\sum_{j=0}^{i} v_{j}^{\alpha} \psi\left(x_{i-j}, t\right)-\sum_{j=0}^{i+1} v_{j}^{\alpha} \psi\left(x_{i-j+1}, t\right)\right] \\
& +\frac{\rho}{4 h^{2}}\left[\psi\left(x_{i-2}, t\right)-2 \psi\left(x_{i}, t\right)+\psi\left(x_{i+2}, t\right)\right] .
\end{aligned}
$$

Now, define a temporal partition $t_{n}=n \tau, n=0,1, \ldots$, where $\tau$ is the time step, and use the standard backward difference to approximate the temporal derivative in Eq. (36) such that

$$
\left.\frac{d \psi\left(x_{i}, t\right)}{d t}\right|_{t=t_{n+1}}=\frac{\psi\left(x_{i}, t_{n+1}\right)-\psi\left(x_{i}, t_{n}\right)}{\tau}+\mathcal{O}(\tau) .
$$

Let $\psi_{i}^{n} \approx \psi\left(x_{i}, t_{n}\right)$ denote the numerical solution. Then we have

$$
\begin{aligned}
\frac{\psi_{i}^{n+1}-\psi_{i}^{n}}{\tau}= & \epsilon h^{\alpha-1}\left[\sum_{j=0}^{i} v_{j}^{\alpha} \psi_{i-j}^{n+1}-\sum_{j=0}^{i+1} v_{j}^{\alpha} \psi_{i-j+1}^{n+1}\right] \\
& +\frac{\rho}{4 h^{2}}\left[\psi_{i-2}^{n+1}-2 \psi_{i}^{n+1}+\psi_{i+2}^{n+1}\right]
\end{aligned}
$$

By collecting like terms, we rewrite Eq. (37) in the form

$$
\frac{\psi_{i}^{n+1}-\psi_{i}^{n}}{\tau}=\frac{1}{h} \sum_{j=0}^{N} k_{i j} \psi_{j}^{n+1}, \quad i=0,1,2, \ldots, N
$$

where $k_{i j}$ is given by

$$
k_{i j}= \begin{cases}\epsilon h^{\alpha}\left[v_{i-j}^{\alpha}-v_{i-j+1}^{\alpha}\right], & j<i-2, \\ \epsilon h^{\alpha}\left[v_{2}^{\alpha}-v_{3}^{\alpha}\right]+\frac{\rho}{4 h}, & j=i-2, \\ \epsilon h^{\alpha}\left[v_{1}^{\alpha}-v_{2}^{\alpha}\right], & j=i-1, \\ \epsilon h^{\alpha}\left[v_{0}^{\alpha}-v_{1}^{\alpha}\right]-\frac{\rho}{2 h}, & j=i, \\ \epsilon h^{\alpha}\left[-v_{0}^{\alpha}\right], & j=i+1, \\ \frac{\rho}{4 h}, & j=i+2, \\ 0, & j>i+2 .\end{cases}
$$


The vector of the numerical solution $\psi^{n}=\left[\psi_{0}^{n}, \psi_{1}^{n}, \ldots, \psi_{N}^{n}\right]$ can be denoted by

$$
\left(I+\frac{\tau}{h} A\right) \psi^{n+1}=\psi^{n}
$$

where the matrix $A$ has the elements $a_{i j}=-k_{i j}$.

The next two theorems explain that the finite volume scheme described in Eq. (37) is conditionally stable and consistent with first-order accuracy in time and second-order accuracy in space.

Theorem 5 For $i=0,1, \ldots, N$, the numerical scheme $\frac{\psi_{i}^{n+1}-\psi_{i}^{n}}{\tau}=\frac{1}{h} \sum_{j=0}^{N} k_{i j} \psi_{j}^{n+1}$ is conditionally stable.

Proof Substitute $\psi_{i}^{n}=\hat{\psi}^{n} \exp (i I \xi)$ into the numerical scheme to get

$$
\frac{\psi_{i}^{n+1}-\psi_{i}^{n}}{\tau}=\frac{1}{h} \sum_{j=0}^{N} k_{i j} \psi_{j}^{n+1}, \quad i=0,1,2, \ldots, N
$$

However, we have

$$
\hat{\psi}^{n+1} \exp (i I \xi)-\psi^{n} \exp (i I \xi)=r \sum_{j=0}^{N} k_{i j} \hat{\psi}^{n+1} \exp (j I \xi), \quad r=\frac{\tau}{h}, \hat{\psi}^{n+1}=\rho(\xi) \hat{\psi}^{n}
$$

where

$$
\rho(\xi)=\frac{1}{\left[1-r \sum_{j=0}^{N} k_{i j} \exp ((j-i) I \xi)\right]}
$$

satisfies the von Neumann condition when $\left|1-r \sum_{j=0}^{N} k_{i j} \exp ((j-i) I \xi)\right| \geq 1$. Therefore, by using the reverse triangle inequality, we have

$$
\left|1-r \sum_{j=0}^{N} k_{i j} \exp ((j-i) I \xi)\right| \geq|1-| r \sum_{j=0}^{N} k_{i j} \exp ((j-i) I \xi)|| .
$$

Hence, the von Neumann condition will be satisfied whenever

$$
|1-| r \sum_{j=0}^{N} k_{i j} \exp ((j-i) I \xi)|| \geq 1 .
$$

That is, either

$$
1-\left|r \sum_{j=0}^{N} k_{i j} \exp ((j-i) I \xi)\right| \geq 1,
$$

which is impossible to hold, or

$$
1-\left|r \sum_{j=0}^{N} k_{i j} \exp ((j-i) I \xi)\right| \leq-1,
$$

which is equivalent to $\left|\sum_{j=0}^{N} k_{i j} \exp ((j-i) I \xi)\right| \geq \frac{2}{r}$. 
Thus, the symbol $\rho(\xi)$ of the numerical scheme satisfies the von Neumann condition if

$$
\left|\sum_{j=0}^{N} k_{i j} \exp ((j-i) I \xi)\right| \geq \frac{2}{r}, \quad \forall i=0,1,2, \ldots, N
$$

which means that the numerical scheme is conditionally stable. This completes the proof of the theorem.

Theorem 6 Numerical Scheme (37) is consistent with second-order accuracy in space and first-order one in time.

Proof Insert $\psi(x, t)$ into expression (37) to get

$$
\begin{aligned}
\frac{\psi\left(x_{i}, t_{n+1}\right)-\psi\left(x_{i}, t_{n}\right)}{\tau} & \\
= & \epsilon h^{\alpha-1}\left[\sum_{j=0}^{i} v_{j}^{\alpha} \psi\left(x_{i-j}, t_{n+1}\right)-\sum_{j=0}^{i+1} v_{j}^{\alpha} \psi\left(x_{i-j+1}, t_{n+1}\right)\right] \\
& +\frac{\rho}{4 h^{2}}\left[\psi\left(x_{i-2}, t_{n+1}\right)-2 \psi\left(x_{i-2}, t_{n+1}\right)+\psi\left(x_{i+2}, t_{n+1}\right)\right] \\
T_{i}^{n}= & \frac{\psi\left(x_{i}, t_{n+1}\right)-\psi\left(x_{i}, t_{n}\right)}{\tau}-\epsilon h^{\alpha-1}\left[\sum_{j=0}^{i} v_{j}^{\alpha} \psi\left(x_{i-j}, t_{n+1}\right)-\sum_{j=0}^{i+1} v_{j}^{\alpha} \psi\left(x_{i-j+1}, t_{n+1}\right)\right] \\
& -\frac{\rho}{4 h^{2}}\left[\psi\left(x_{i-2}, t_{n+1}\right)-2 \psi\left(x_{i-2}, t_{n+1}\right)+\psi\left(x_{i+2}, t_{n+1}\right)\right] .
\end{aligned}
$$

By the control volume averages, combined with a temporal backward difference for the time derivative,

$$
\frac{d \bar{\psi}_{i}\left(t_{n+1}\right)}{d t}=\frac{\psi\left(x_{i}, t_{n+1}\right)-\psi\left(x_{i}, t_{n}\right)}{\tau}+\mathcal{O}\left(\tau+h^{2}\right)
$$

we have

$$
\begin{aligned}
T_{i}^{n}= & \frac{d \bar{\psi}_{i}\left(t_{n+1}\right)}{d t}-\mathcal{O}\left(\tau+h^{2}\right)-\epsilon h^{\alpha-1}\left[\sum_{j=0}^{i} v_{j}^{\alpha} \psi_{i-j}^{n+1}-\sum_{j=0}^{i+1} v_{j}^{\alpha} \psi_{i-j+1}^{n+1}\right] \\
& -\frac{v}{4 h^{2}}\left[\psi_{i-2}^{n+1}-2 \psi_{i}^{n+1}+\psi_{i+2}^{n+1}\right] .
\end{aligned}
$$

Substitute (25), (27), and (30) above, it turns to $T_{i}^{n}=\mathcal{O}\left(\tau+h^{2}\right)$. Hence, $T_{i}^{n} \rightarrow 0$ as $\tau \rightarrow 0$ and $h \rightarrow 0$. So, the given numerical scheme is consistent with second-order accuracy in space and first-order in time. This completes the proof of the theorem.

Corollary 3 Numerical Scheme (37) is consistent and stable.

By the fundamental theorem of numerical methods for differential equations, the given numerical scheme is convergent. 
Table 1 Numerical results for Example 1 with varying $\beta$ at time $t=1$, fixed $h=0.015625$ and $\tau=0.01$ using FDSF and FVSF

\begin{tabular}{|c|c|c|c|c|c|c|}
\hline & \multicolumn{2}{|l|}{$\beta=0.7$} & \multicolumn{2}{|l|}{$\beta=0.8$} & \multicolumn{2}{|l|}{$\beta=0.9$} \\
\hline & $\overline{F D S F}$ & FVSF & FDSF & FVSF & FDSF & FVSF \\
\hline 1. & $2.8695 \times 10^{-4}$ & $3.2270 \times 10^{-4}$ & $3.0669 \times 10^{-4}$ & $2.2835 \times 10^{-4}$ & $1.2079 \times 10^{-4}$ & $4.0124 \times 10^{-5}$ \\
\hline 1.015625 & $5.7376 \times 10^{-4}$ & $6.4525 \times 10^{-4}$ & $6.1325 \times 10^{-4}$ & $4.5659 \times 10^{-4}$ & $2.4151 \times 10^{-4}$ & $8.0218 \times 10^{-5}$ \\
\hline 1.03125 & $8.6030 \times 10^{-4}$ & $9.6750 \times 10^{-4}$ & $9.1951 \times 10^{-4}$ & $6.8460 \times 10^{-4}$ & $3.6208 \times 10^{-4}$ & $12.0254 \times 10^{-5}$ \\
\hline 1.046875 & $1.14637 \times 10^{-3}$ & $12.8924 \times 10^{-4}$ & $1.22528 \times 10^{-3}$ & $9.1221 \times 10^{-4}$ & $4.8242 \times 10^{-4}$ & $16.0196 \times 10^{-5}$ \\
\hline 1.0625 & $1.43177 \times 10^{-3}$ & $16.1023 \times 10^{-4}$ & $1.53032 \times 10^{-3}$ & $11.3925 \times 10^{-4}$ & $6.0243 \times 10^{-4}$ & $20.0010 \times 10^{-5}$ \\
\hline 1.078125 & $7 \times 10^{-3}$ & $19.3022 \times 10^{-4}$ & $1.83439 \times 10^{-3}$ & $13.6553 \times 10^{-4}$ & $7.2200 \times 10^{-4}$ & 23.966 \\
\hline 1.09375 & $4 \times 10^{-3}$ & $22.4893 \times 10^{-4}$ & $2.13724 \times 10^{-3}$ & $15.9087 \times 10^{-4}$ & $8.4103 \times 10^{-4}$ & 27.91 \\
\hline 109375 & $10^{-3}$ & 25.661 & $2.43861 \times 10^{-3}$ & $18.1505 \times 10^{-4}$ & $9.5941 \times 10^{-4}$ & $31.8321 \times 10^{-5}$ \\
\hline 1.125 & $2.56202 \times 10^{-3}$ & $28.8149 \times 10^{-4}$ & $2.73823 \times 10^{-3}$ & $20.3789 \times 10^{-4}$ & $10.7703 \times 10^{-4}$ & $35.7259 \times 10^{-5}$ \\
\hline 1.140625 & $2.84055 \times 10^{-3}$ & $31.9476 \times 10^{-4}$ & $3.03583 \times 10^{-3}$ & $22.5918 \times 10^{-4}$ & $11.9379 \times 10^{-4}$ & $39.5886 \times 10^{-5}$ \\
\hline 1.15625 & $3.11696 \times 10^{-3}$ & $35.0567 \times 10^{-4}$ & $3.33115 \times 10^{-3}$ & $24.7871 \times 10^{-4}$ & $13.0957 \times 10^{-4}$ & $43.4166 \times 10^{-5}$ \\
\hline 1.171875 & $3.39102 \times 10^{-3}$ & $38.1392 \times 10^{-4}$ & $3.62391 \times 10^{-3}$ & $26.9629 \times 10^{-4}$ & $14.2428 \times 10^{-4}$ & $47.2062 \times 10^{-5}$ \\
\hline 1.1875 & $3.66247 \times 10$ & $41.1923 \times 10^{-4}$ & $3.91384 \times 10^{-3}$ & $29.1171 \times 10^{-4}$ & $15.3779 \times 10^{-4}$ & $50.9538 \times 10^{-5}$ \\
\hline 1.203125 & $3.93106 \times 10^{-3}$ & $44.2132 \times 10^{-4}$ & $4.20068 \times 10^{-3}$ & $31.2477 \times 10^{-4}$ & $16.5000 \times 10^{-4}$ & $54.6558 \times 10^{-5}$ \\
\hline 1.21875 & $4.19653 \times 10^{-3}$ & $47.1990 \times 10^{-4}$ & $4.48415 \times 10^{-3}$ & $33.3527 \times 10^{-4}$ & $17.6081 \times 10^{-4}$ & $58.3087 \times 10^{-5}$ \\
\hline 1.234375 & & $50.14697 \times 10^{-4}$ & $4.76397 \times 10^{-3}$ & $35.4299 \times 10^{-4}$ & $18.7011 \times 10^{-4}$ & $61.9088 \times 10^{-5}$ \\
\hline 1.25 & $4.71715 \times 10^{-3}$ & $53.0542 \times 10^{-4}$ & $5.03990 \times 10^{-3}$ & $37.4776 \times 10^{-4}$ & $19.7778 \times 10^{-4}$ & $65.4528 \times 10^{-5}$ \\
\hline
\end{tabular}

\section{Numerical experiments}

In this section, we introduce the numerical problems to verify the correctness and effectiveness of the proposed schemes. Both schemes can efficiently be solved, but the FVSF is more accurate than the FDSF. Two examples are introduced by comparing the numerical solutions with the exact solutions. The computations are performed by Mathematica Software 11.0.

Example 1 Consider the following SFCDE:

$$
\frac{\partial u(x, t)}{\partial t}+\epsilon \frac{\partial^{\beta} u(x, t)}{\partial x^{\beta}}=\rho \frac{\partial^{2} u(x, t)}{\partial x^{2}}
$$

subject to the initial condition

$$
u(x, 0)=-\sin (\pi x),
$$

where $x \in[1,2.5], t \geq 0, \epsilon=1, \rho=2$, and $0<\beta \leq 1$. For $\beta=1$, the exact solution is $u(x, t)=-\sin (\pi(x-t)) \exp \left(-2\left(\pi^{2} t\right)\right)$. The proposed schemes are applied to solve the above problem for different values of $\beta$ such that $\beta \in\{0.7,0.8,0.9,1\}$, by fixed $h=0.015625$ and $\tau=0.01, h$ and $\tau$ are chosen small enough to demonstrate the accuracy of our schemes. In Table 1 , we show the numerical results at $t=1$ with varying $\beta$ such that $\beta \in\{0.7,0.8,0.9\}$ and $x \in[1,1.25]$ using the FDSF and the FVSF; while the absolute errors between the numerical solution in both schemes and the exact solution are reported in Table 2. When $\beta=1, t=1$, and $x \in[1,1.25]$, this table demonstrates that there is an agreement between the approximate solution and the exact solution, which confirms that the proposed schemes are effective. According to our observations, the FVSF is more accurate than the FDSF. The 2D plot of the solutions behavior with varying $\beta$ such that $\beta \in\{0.7,0.8,0.9\}$ is drawn in Fig. 1 at $t=1$ and $x \in[1,2.5]$ using the FVSF and the FDSF; while Fig. 2 shows 
Table 2 Exact solution and absolute errors for Example 1 at time $t=1, \beta=1$, fixed $h=0.015625$ and $\tau=0.01$ using FDSF and FVSF

\begin{tabular}{llll}
\hline$x$ & Exact solution & Absolute error & \\
\cline { 3 - 3 } & & FDSF & Absolute error \\
\hline 1. & 0. & $7.193791 \times 10^{-5}$ & $5.814751 \times 10^{-6}$ \\
1.015625 & $-1.312701 \times 10^{-10}$ & $1.438076 \times 10^{-5}$ & $1.162322 \times 10^{-7}$ \\
1.03125 & $-2.622240 \times 10^{-10}$ & $2.1553847 \times 10^{-5}$ & $1.741653 \times 10^{-7}$ \\
1.046875 & $-3.925462 \times 10^{-10}$ & $2.8706087 \times 10^{-5}$ & $2.318803 \times 10^{-7}$ \\
1.0625 & $-5.219227 \times 10^{-10}$ & $3.5830568 \times 10^{-5}$ & $2.893150 \times 10^{-7}$ \\
1.078125 & $-6.500419 \times 10^{-10}$ & $4.2920399 \times 10^{-5}$ & $3.464091 \times 10^{-7}$ \\
1.09375 & $-7.765951 \times 10^{-10}$ & $4.9968719 \times 10^{-5}$ & $4.031040 \times 10^{-7}$ \\
1.109375 & $-9.012773 \times 10^{-10}$ & $5.696871 \times 10^{-5}$ & $4.593420 \times 10^{-7}$ \\
1.125 & $-1.023788 \times 10^{-9}$ & $6.391360 \times 10^{-5}$ & $5.150668 \times 10^{-7}$ \\
1.140625 & $-1.143833 \times 10^{-9}$ & $7.079666 \times 10^{-5}$ & $5.702229 \times 10^{-7}$ \\
1.15625 & $-1.261122 \times 10^{-9}$ & $7.761125 \times 10^{-5}$ & $6.247557 \times 10^{-7}$ \\
1.171875 & $-1.375372 \times 10^{-9}$ & $8.435077 \times 10^{-5}$ & $6.786116 \times 10^{-7}$ \\
1.1875 & $-1.486310 \times 10^{-9}$ & $9.100869 \times 10^{-5}$ & $7.317379 \times 10^{-7}$ \\
1.203125 & $-1.593667 \times 10^{-9}$ & $9.757858 \times 10^{-5}$ & $7.840830 \times 10^{-7}$ \\
1.21875 & $-1.697184 \times 10^{-9}$ & $10.40540 \times 10^{-5}$ & $8.355962 \times 10^{-7}$ \\
1.234375 & $-1.796613 \times 10^{-9}$ & $11.04289 \times 10^{-5}$ & $8.862276 \times 10^{-7}$ \\
1.25 & $-1.891714 \times 10^{-9}$ & $11.66969 \times 10^{-5}$ & $9.359287 \times 10^{-7}$ \\
\hline
\end{tabular}

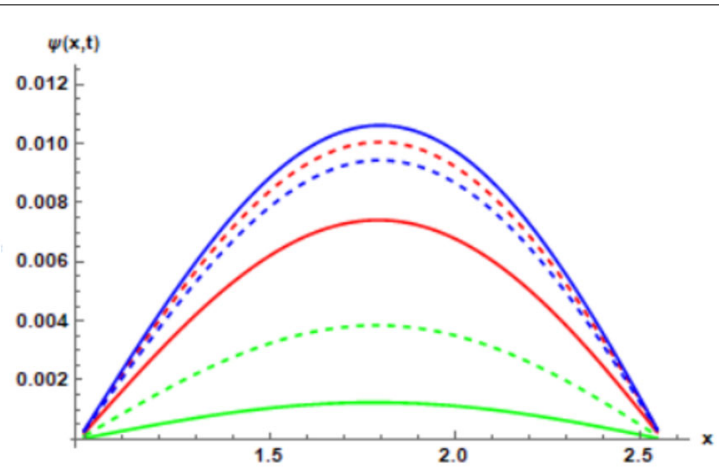

FDSF $\beta=0.90$

- FVSF $\beta=0.90$

FDSF $\beta=0.80$

— FVSF $\beta=0.80$

FDSF $\beta=0.70$

FVSF $\beta=0.70$

Figure 1 Solutions to Example 1 at time $t=1$ for varying $\beta$ using FDSF and FVSF

that FVSF provides an excellent agreement with the exact solution rather than the FDSF. Moreover, the 3D plot of the exact solution behavior and the solutions behavior using the FVSF is drawn in Fig. 3 at $\beta=1, t \in[0,1]$ and $x \in[1,2.5]$.

Example 2 Consider the following SFCDE:

$$
\frac{\partial u(x, t)}{\partial t}+\epsilon \frac{\partial^{\beta} u(x, t)}{\partial x^{\beta}}=\rho \frac{\partial^{2} u(x, t)}{\partial x^{2}},
$$

subject to the initial condition

$$
u(x, 0)=e^{\zeta x},
$$

where $x \in[-2,4], \epsilon=0.1, \rho=0.02, t \geq 0,0<\beta \leq 1$, and $\zeta=1.1771243444677$. For $\beta=1$, the exact solution is $u(x, t)=e^{\zeta x-m t}, m=0.09$. The FDSF and FVSF are tested numerically to solve the above problem for different values of $\beta$ such that $\beta \in\{0.5,0.7,0.9,1\}$, by 


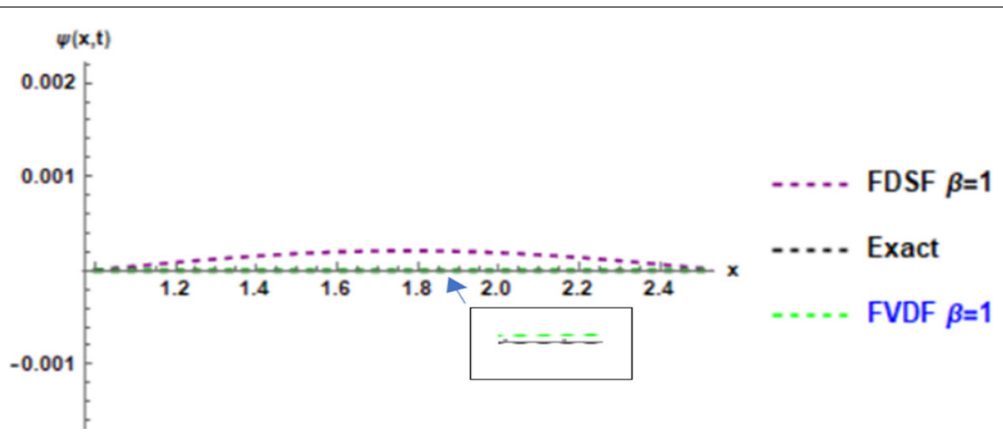

Figure 2 Exact and approximate solutions to Example 1 at time $t=1$ for $\beta=1$ using FDSF and FVSF. Here black and green lines coincide to some extent
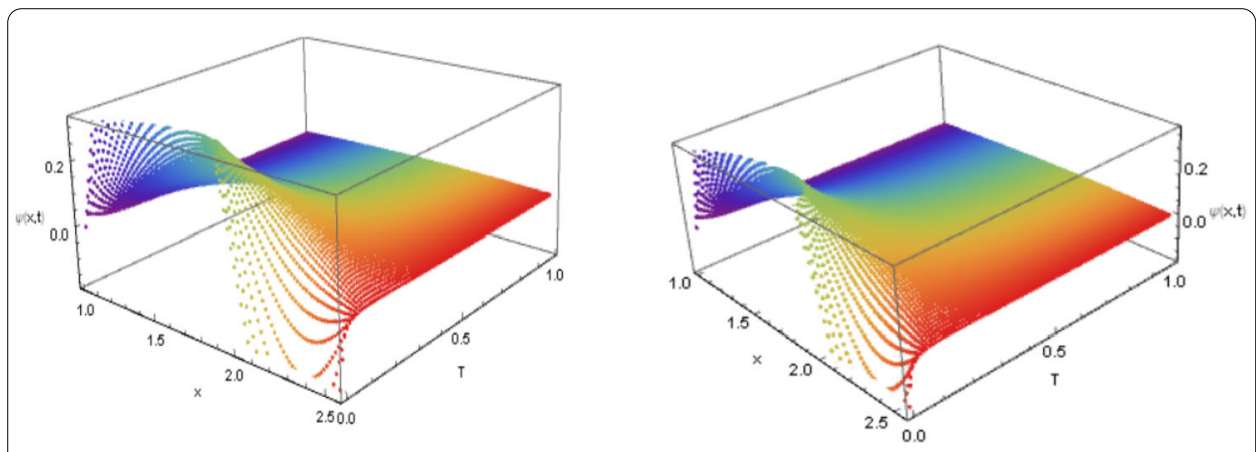

Figure 3 Surface plot to Example 1 at $\beta=1$ exact solution (left) and using FVSF (right)

fixed $h=0.0625$ and $\tau=0.01$. Here, $h$ and $\tau$ are chosen small enough to demonstrate the accuracy of our schemes. The numerical results at $t=1$ with varying $\beta$ such that $\beta \in\{0.5,0.7,0.9\}$ and $x \in[-2,-1.25]$ using the FDSF and the FVSF are reported in Table 3. When $\beta=1, t=1$, and $x \in[-2,-1.25]$, the numerical results are reported in Table 4 . These results demonstrate that the FVSF is more significantly accurate than the FDSF, and it can be observed through by the absolute errors between the numerical solution in both schemes and the exact solution. The 2D plot of the solutions behavior with varying $\beta$ such that $\beta \in\{0.5,0.7,0.90\}$ is drawn in Fig. 4 at $t=1$ and $x \in[-2,1]$ using the FDSF and the FVSF, while Fig. 5 shows that the FVSF provides an excellent agreement with the exact solution rather than the FDSF. Moreover, the 3D plot of the exact solution behavior and the solutions behavior using the FVSF is drawn in Fig. 3 at $\beta=1, t \in[0,1]$, and $x \in[-2,1]$. Figure 6 shows the surface plot of the solution behavior at $\beta=1$ by using the FVSF and the FDSF at $t \in[0,1]$ and $x \in[-2,4]$.

Table 3 and Table 4 depict that the solution obtained by the FVSF is more accurate and closer to the exact solution compared with the solution obtained by the FDSF in two different experiments. Also, it is easy to increase the nodes in the regular grid to get higherorder accuracy. Thus, it can be concluded that the FVM is effective for solving the SFCDE with a constant coefficient. This implicit scheme can be improved for implementation when tackling differential equations, in specific for problems with or without source terms that are deformed or nonlinear. Also, it can handle general condition without difficulty and can deal with unlimited kinds of the initial condition. 
Table 3 Numerical results for Example 2 with varying $\beta$ at time $t=1$, fixed $h=0.0625$ and $\tau=0.01$ using FDSF and FVSF

\begin{tabular}{|c|c|c|c|c|c|c|}
\hline \multirow[t]{2}{*}{$x$} & \multicolumn{2}{|l|}{$\beta=0.7$} & \multicolumn{2}{|l|}{$\beta=0.8$} & \multicolumn{2}{|l|}{$\beta=0.9$} \\
\hline & FDSF & FVSF & FDSF & FVSF & FDSF & FVSF \\
\hline-2. & 0.030621 & 0.030618 & 0.030579 & 0.030491 & 0.03033986 & 0.03033981 \\
\hline-1.9375 & 0.059504 & 0.059501 & 0.059414 & 0.059216 & 0.05887638 & 0.05887635 \\
\hline-1.875 & 0.085525 & 0.085520 & 0.085364 & 0.085010 & 0.08439982 & 0.08439979 \\
\hline-1.8125 & 0.108188 & 0.108174 & 0.107925 & 0.107367 & 0.10641620 & 0.10641618 \\
\hline-1.75 & 0.127745 & 0.127712 & 0.127348 & 0.126555 & 0.12521788 & 0.12521786 \\
\hline-1.6875 & 0.144948 & 0.144883 & 0.144390 & 0.143344 & 0.14160306 & 0.14160304 \\
\hline-1.625 & 0.160711 & 0.160604 & 0.159973 & 0.158670 & 0.15652714 & 0.15652713 \\
\hline-1.5625 & 0.175875 & 0.175718 & 0.174945 & 0.173386 & 0.17085273 & 0.17085271 \\
\hline-1.5 & 0.191093 & 0.190881 & 0.189964 & 0.188154 & 0.18524192 & 0.18524190 \\
\hline-1.4375 & 0.206829 & 0.206559 & 0.205497 & 0.203438 & 0.20015484 & 0.20015483 \\
\hline-1.375 & 0.223397 & 0.223067 & 0.221860 & 0.219551 & 0.21589775 & 0.21589774 \\
\hline-1.3125 & 0.241017 & 0.240626 & 0.239269 & 0.236708 & 0.23267882 & 0.23267880 \\
\hline-1.25 & 0.259854 & 0.259399 & 0.257890 & 0.255068 & 0.25065218 & 0.25065216 \\
\hline
\end{tabular}

Table 4 Exact solution and absolute errors for Example 2 at time $t=1, \beta=1$, fixed $h=0.0625$ and $\tau=0.01$

\begin{tabular}{llll}
\hline$x$ & Exact solution & Absolute error & Absolute error \\
& & FDSF & FVSF \\
\hline-2. & 0.02949131 & $2.860725 \times 10^{-4}$ & $8.30618 \times 10^{-5}$ \\
-1.9375 & 0.05741732 & $1.941801 \times 10^{-4}$ & $9.50135 \times 10^{-5}$ \\
-1.875 & 0.08254919 & $4.107925 \times 10^{-4}$ & $8.55201 \times 10^{-5}$ \\
-1.8125 & 0.10222554 & $6.965656 \times 10^{-4}$ & $1.08174 \times 10^{-5}$ \\
-1.75 & 0.12048793 & $1.199878 \times 10^{-4}$ & $1.27712 \times 10^{-5}$ \\
-1.6875 & 0.13548110 & $1.046201 \times 10^{-4}$ & $4.48834 \times 10^{-5}$ \\
-1.625 & 0.14885322 & $1.139510 \times 10^{-4}$ & $6.06044 \times 10^{-5}$ \\
-1.5625 & 0.16225611 & $2.306122 \times 10^{-4}$ & $7.57187 \times 10^{-5}$ \\
-1.5 & 0.17534557 & $1.393114 \times 10^{-4}$ & $9.08811 \times 10^{-5}$ \\
-1.4375 & 0.18828164 & $6.647887 \times 10^{-4}$ & $0.20690 \times 10^{-5}$ \\
-1.375 & 0.20312896 & $3.956443 \times 10^{-4}$ & $0.70671 \times 10^{-5}$ \\
-1.3125 & 0.21995710 & $8.339676 \times 10^{-4}$ & $0.84062 \times 10^{-5}$ \\
-1.25 & 0.23504094 & $8.336256 \times 10^{-4}$ & $0.25998 \times 10^{-5}$ \\
\hline
\end{tabular}

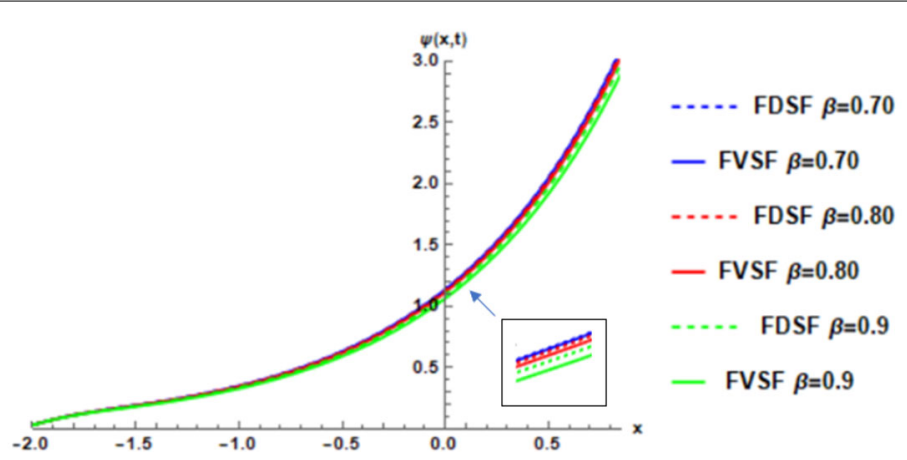

Figure 4 Solutions to Example 2 at time $t=1$ for varying $\beta$ using FDSF and FVSF

\section{Conclusions}

The SFCDE is one of the most important equations used to describe physical phenomena. In this paper, the FDM and the FVM have been developed for solving the SFCDE. The space fractional derivative in this equation was considered in the Riemann-Liouville 


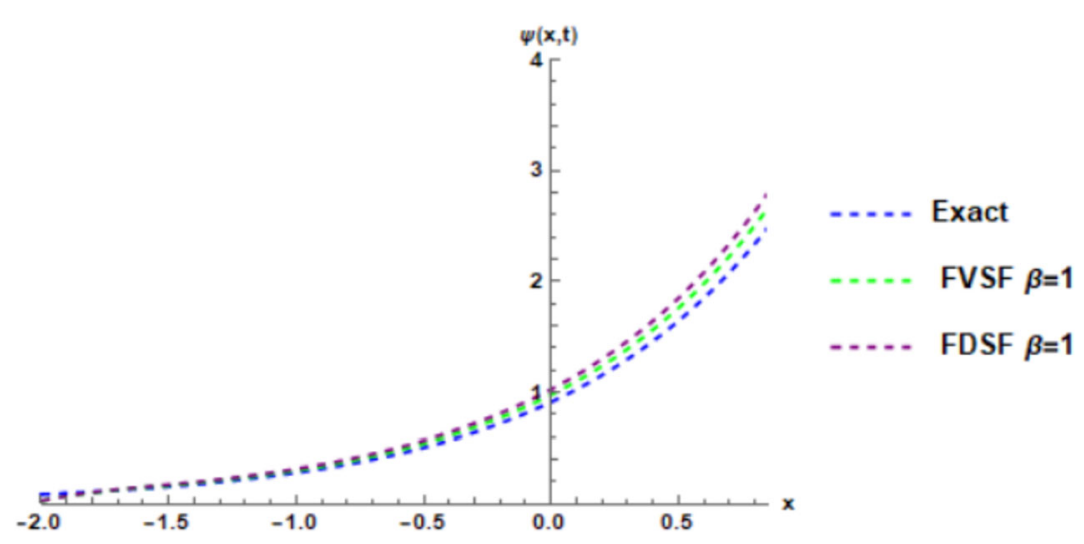

Figure 5 Exact and approximate solutions using FDSF and FVSF for Example 2 at time $t=1$ and $\beta=1$

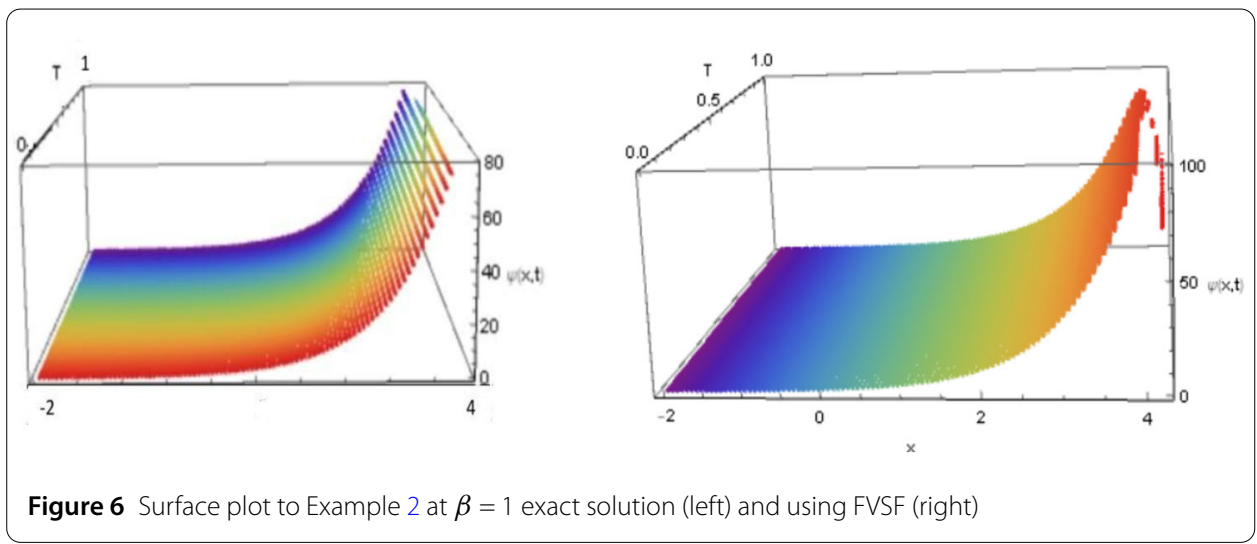

sense. The Grünwald-Letnikov fractional formula has been used for the discretization of space fractional derivative, and in both formulations the implicit scheme has been introduced. It has been shown that both schemes are stable with first-order accuracy in time and second-order accuracy in space. Numerical results have been found using Mathematica software 11.0. Comparing illustrated examples justified the efficiency and accuracy of the proposed methods.

\section{Acknowledgements}

The authors gratefully acknowledge the support of Springer Nature.

Funding

This work was gratefully supported by the Springer Nature.

Availability of data and materials

Please contact the authors for data requests.

\section{Declarations}

Competing interests

Authors declare that they have no competing interests regarding the publication of the article.

\section{Authors' contributions}

The authors contributed equally and significantly in writing this paper. All authors read and approved the final manuscript. 


\section{Author details}

'Department of Mathematics, Taibah University, Al Madinah Al Munawara, Saudi Arabia. ${ }^{2}$ Department of Physics and Basic Sciences, Faculty of Engineering Technology, Al-Balqa Applied University, Amman, Jordan. ${ }^{3}$ Department of Applied Science, Ajloun College, Al-Balqa Applied University, Ajloun, 26816, Jordan. ${ }^{4}$ Nonlinear Dynamics Research Center (NDRC), Ajman University, Ajman, UAE. ${ }^{5}$ Department of Mathematics, Faculty of Science, University of Jordan, Amman, 11942, Jordan. ${ }^{6}$ Department of Mathematics and Computer Sciences, Universitatea Transilvania Brasov, Brasov, Romania.

\section{Publisher's Note}

Springer Nature remains neutral with regard to jurisdictional claims in published maps and institutional affiliations.

Received: 12 July 2021 Accepted: 29 September 2021 Published online: 29 November 2021

\section{References}

1. Agrawal, O.P.: Solution for a fractional diffusion-wave equation defined in a bounded domain. Nonlinear Dyn. 29(1-4), 145-155 (2002)

2. Chen, Y., Wu, Y., Cui, Y., Wang, Z., Jin, D.: Wavelet method for a class of fractional convection-diffusion equation with variable coefficients. J. Comput. Sci. 1(3), 146-149 (2010)

3. Abu Arqub, O., Edwan, R., Al-Smadi, M., Momani, S.: Solving space-fractional Cauchy problem by modified finite-difference discretization scheme. Alex. Eng. J. 59(4), 2409-2417 (2020)

4. Shou, D.-H., He, J.-H.: Beyond Adomian method: the variational iteration method for solving heat-like and wave-like equations with variable coefficients. Phys. Lett. A 372(3), 233-237 (2008)

5. Faminskii, A.V.: An initial boundary-value problem in a half-strip for the Korteweg-de Vries equation in fractional-order Sobolev spaces. Commun. Partial Differ. Equ. 29(11-12), 1653-1695 (2005)

6. Al-Smadi, M., Dutta, H., Hasan, S., Momani, S.: On numerical approximation of Atangana-Baleanu-Caputo fractional integro-differential equations under uncertainty in Hilbert space. Math. Model. Nat. Phenom. 16, 030 (2021)

7. Al-Smadi, M., Djeddi, N., Momani, S., Al-Omari, S., Araci, S.: An attractive numerical algorithm for solving nonlinear Caputo-Fabrizio fractional Abel differential equation in a Hilbert space. Adv. Differ. Equ. 2021, Article ID 271 (2021)

8. Al-Smadi, M., Freihat, A., Khalil, H., Momani, S., Khan, R.A.: Numerical multistep approach for solving fractional partial differential equations. Int. J. Comput. Methods 14(3), 1750029 (2017)

9. Al-Smadi, M., Abu Arqub, O., Zeidan, D.: Fuzzy fractional differential equations under the Mittag-Leffler kernel differential operator of the ABC approach: theorems and applications. Chaos Solitons Fractals 146, 110891 (2021)

10. Al-Smadi, M., Arqub, O.A., Shawagfeh, N., Momani, S.: Numerical investigations for systems of second-order periodic boundary value problems using reproducing kernel method. Appl. Math. Comput. 291, 137-148 (2016)

11. Al-Smadi, M., Abu Arqub, O., Gaith, M.: Numerical simulation of telegraph and Cattaneo fractional-type models using adaptive reproducing kernel framework. Math. Methods Appl. Sci. 44(10), 8472-8489 (2021)

12. Al-Smadi, M., Abu Arqub, O.: Computational algorithm for solving Fredholm time-fractional partial integrodifferential equations of Dirichlet functions type with error estimates. Appl. Math. Comput. 342, 280-294 (2019)

13. Abu Arqub, O., Al-Smadi, M.: An adaptive numerical approach for the solutions of fractional advection-diffusion and dispersion equations in singular case under Riesz's derivative operator. Phys. A, Stat. Mech. Appl. 540, 123257 (2020)

14. Al-Smadi, M.: Simplified iterative reproducing kernel method for handling time-fractional BVPs with error estimation. Ain Shams Eng. J. 9(4), 2517-2525 (2018)

15. Hasan, S., Al-Smadi, M., El-Ajou, A., Momani, S., Hadid, S., Al-Zhour, Z.: Numerical approach in the Hilbert space to solve a fuzzy Atangana-Baleanu fractional hybrid system. Chaos Solitons Fractals 143, 110506 (2021)

16. Al-Smadi, M., Abu Arqub, O., Momani, S.: Numerical computations of coupled fractional resonant Schrödinger equations arising in quantum mechanics under conformable fractional derivative sense. Phys. Scr. 95(7), 075218 (2020)

17. Al-Smadi, M., Abu Arqub, O., Hadid, S.: An attractive analytical technique for coupled system of fractional partial differential equations in shallow water waves with conformable derivative. Commun. Theor. Phys. 72(8), 085001 (2020)

18. Al-Smadi, M., Abu Arqub, O., Hadid, S.: Approximate solutions of nonlinear fractional Kundu-Eckhaus and coupled fractional massive Thirring equations emerging in quantum field theory using conformable residual power series method. Phys. Scr. 95(10), 105205 (2020)

19. Momani, S., Odibat, Z.: Homotopy perturbation method for nonlinear partial differential equations of fractional order Phys. Lett. A 365(5-6), 345-350 (2007)

20. Momani, S., Djeddi, N., Al-Smadi, M., Al-Omari, S.: Numerical investigation for Caputo-Fabrizio fractional Riccati and Bernoulli equations using iterative reproducing kernel method. Appl. Numer. Math. 170, 418-434 (2021)

21. Adigüzel, R.S., Aksoy, Ü., Karapinar, E., Erhan, I.M.: On the solution of a boundary value problem associated with a fractional differential equation. Math. Methods Appl. Sci. (2020). https://doi.org/10.1002/mma.6652

22. Afshari, H., Kalantari, S., Baleanu, D.: Solution of fractional differential equations via $\alpha-\psi$-Geraghty type mappings. Adv. Differ. Equ. 2018, Article ID 347 (2018)

23. Afshari, H., Karapınar, E.: A discussion on the existence of positive solutions of the boundary value problems via $\psi$-Hilfer fractional derivative on b-metric spaces. Adv. Differ. Equ. 2020, Article ID 616 (2020)

24. Afshari, H., Baleanu, D.: Applications of some fixed point theorems for fractional differential equations with Mittag-Leffler kernel. Adv. Differ. Equ. 2020, Article ID 140 (2020)

25. Izadkhah, M.M., Saberi-Nadjafi, J.: Gegenbauer spectral method for time-fractional convection-diffusion equations with variable coefficients. Math. Methods Appl. Sci. 38(15), 3183-3194 (2015)

26. Saadatmandi, A., Dehghan, M., Azizi, M.-R.: The Sinc-Legendre collocation method for a class of fractional convection-diffusion equations with variable coefficients. Commun. Nonlinear Sci. Numer. Simul. 17(11), 4125-4136 (2012)

27. Karaa, S., Mustapha, K., Pani, A.K.: Finite volume element method for two-dimensional fractional subdiffusion problems. IMA J. Numer. Anal. 37(2), 945-964 (2017) 
28. Jia, J., Wang, H.: A fast finite volume method for conservative space-fractional diffusion equations in convex domains. J. Comput. Phys. 310, 63-84 (2016)

29. Mahto, L., Abbas, S., Hafayed, M., Srivastava, H.M.: Approximate controllability of sub-diffusion equation with impulsive condition. Mathematics 7(2), 190 (2019)

30. Zhao, Y., Zhang, Y., Shi, D., Liu, F., Turner, I.: Superconvergence analysis of nonconforming finite element method for two-dimensional time fractional diffusion equations. Appl. Math. Lett. 59, 38-47 (2016)

31. Zhao, Y., Chen, P., Bu, W., Liu, X., Tang, Y.: Two mixed finite element methods for time-fractional diffusion equations. J. Sci. Comput. 70(1), 407-428 (2017)

32. Stynes, M., O'Riordan, E., Gracia, J.L.: Error analysis of a finite difference method on graded meshes for a time-fractional diffusion equation. SIAM J. Numer. Anal. 55(2), 1057-1079 (2017)

33. Sayevand, K., Arjang, F.: Finite volume element method and its stability analysis for analyzing the behavior of sub-diffusion problems. Appl. Math. Comput. 290, 224-239 (2016)

34. Qiao, L., Xu, D., Wang, Z: An ADI difference scheme based on fractional trapezoidal rule for fractional integro-differential equation with a weakly singular kernel. Appl. Math. Comput. 354, 103-114 (2019)

35. Abdeljawad, A., Agarwal, R.P., Karapinar, E., Kumari, P.S.: Solutions of the nonlinear integral equation and fractiona differential equation using the technique of a fixed point with a numerical experiment in extended b-metric space. Symmetry 11, 686 (2019)

36. Eddine Lazreg, J., Abbas, S., Benchohra, M., Karapinar, E.: Impulsive Caputo-Fabrizio fractional differential equations in b-metric spaces. Open Math. 19, 363-372 (2021)

37. Afshari, H., Kalantari, S., Karapinar, E.: Solution of fractional differential equations via coupled fixed point. Electron. J. Differ. Equ. 2015, Article ID 286 (2015)

38. Alqahtani, B., Aydi, H., Karapinar, E., Rakocevic, V.: A solution for Volterra fractional integral equations by hybrid contractions. Mathematics 2019(7), 694 (2019)

39. Abu-Gdairi, R., Hasan, S., Al-Omari, S., Al-Smadi, M. Momani, S.: Attractive multistep reproducing kernel approach for solving stiffness differential systems of ordinary differential equations and some error analysis. Comput. Model. Eng. Sci. (2021). To appear

40. Gumah, G., Naser, M., Al-Smadi, M., Al-Omari, S.K.: Application of reproducing kernel Hilbert space, method for solving second-order fuzzy Volterra integro-differential equation. Adv. Differ. Equ. 2018, Article ID 475 (2018)

41. Gumah, G., Naser, M., Al-Smadi, M., Al-Omari, S.K., Baleanu, D.: Numerical solutions of hybrid fuzzy differential equations in a Hilbert space. Appl. Numer. Math. 151, 402-412 (2020)

42. Al-Omari, S., Agarwal, P.: Some general properties of a fractional Sumudu transform in the class of Boehmians. Kuwait J. Sci. 43(2), 206-220 (2016)

43. Salim, A., Benchohra, B., Karapinar, E., Lazreg, J.E.: Existence and Ulam stability for impulsive generalized Hilfer-type fractional differential equations. Adv. Differ. Equ. 2020, Article ID 601 (2020)

44. Adiguzel, R.S., Aksoy, U., Karapınar, E., Erhan, I.M.: Uniqueness of solution for higher-order nonlinear fractional differential equations with multi-point and integral boundary conditions. RACSAM 115, Article ID 155 (2021)

45. Adiguzel, R.S., Aksoy, U., Inci, E.K., Erhan, M.: On the solutions of fractional differential equations via Geraghty type hybrid contractions. Appl. Comput. Math. 20(2), 313-333 (2021)

46. Al-Omari, S.: A fractional Fourier integral operator and its extension to classes of function spaces. Adv. Differ. Equ. 2018, 195 (2018)

47. Omari, S., Suthar, D.L., Araci, S.: A fractional q-integral operator associated with certain class of q-Bessel functions and q-generating series. Adv. Differ. Equ. 2021, Article ID 441 (2021)

\section{Submit your manuscript to a SpringerOpen ${ }^{\circ}$ journal and benefit from:}

- Convenient online submission

- Rigorous peer review

- Open access: articles freely available online

- High visibility within the field

- Retaining the copyright to your article

Submit your next manuscript at $\gg$ springeropen.com 